\title{
A MAP OF DEPENDENCIES AMONG THREE-VALUED LOGICS
}

\author{
D. CIUCCI* AND D. DUBOIS $^{O}$
}

\begin{abstract}
Three-valued logics arise in several fields of computer science, both inspired by concrete problems (such as in the management of the null value in databases) and theoretical considerations. Several three-valued logics have been defined. They differ by their choice of basic connectives, hence also from a syntactic and proof-theoretic point of view. Different interpretations of the third truth value have also been suggested. They often carry an epistemic flavor. In this work, relationships between logical connectives on three-valued functions are explored. Existing theorems of functional completeness have laid bare some of these links, based on specific connectives. However we try to draw a map of such relationships between conjunctions, negations and implications that extend Boolean ones. It turns out that all reasonable connectives can be defined from a few of them and so all known three-valued logics appear as a fragment of only one logic. These results can be instrumental when choosing, for each application context, the appropriate fragment where the basic connectives make full sense, based on the appropriate meaning of the third truth-value.
\end{abstract}

Keywords: Three-valued logics, truth-tables, functional completeness.

\section{INTRODUCTION}

Classical Boolean logic is unique in the sense that the definition of its basic connectives is not questionable and there is complete agreement on its model-based semantics. On the contrary, there exists a large body of scattered literature on 3 -valued logics. Formal three-valued logic systems have been studied by various mathematicians, providing axiomatizations in the Hilbert or Gentzen style, and investigating their algebraic properties. There are well-known three-valued logics, like Łukasiewicz [Borowski(1970)] or Kleene [Kleene(1952)], and less known ones like Sobociński's [Sobociński(1952)] and Nelson's [Nelson(1949)]. There seems to be no agreement about what these logics are good for and what should be the definition of their logical connectives. Nevertheless, they turned out to be useful in various areas, as heterogeneous as logic programming [Fitting(1985)], electronic circuits [Smith(1981)] and databases [Codd(1979)], among other topics. Threevalued logics can also be viewed as special kinds of fuzzy logics [Hájek(1998)], where the truth set is a chain of three elements. Fuzzy logics, that often assume the truth-set is infinite, have received considerable attention in the last 15 years. It is thus interesting to go back to the most elementary case not reducible to Boolean logic. As fuzzy logic underlies fuzzy sets, three-valued logics lead to three-valued sets, that can be modeled by pairs of nested sets or orthopairs of disjoint sets

INFORMATION SCIENCES 250, 162-177, 2013, DOI:10.1016/J.INS.2013.06.040 
[Cattaneo and Ciucci(2003), Yao(2012)]. Several kinds of generalized sets can be associated with three-valued logics: rough sets [Ciucci and Dubois(2010)], conditional events [Walker(1994)], shadowed sets [Pedrycz(2009)], interval sets [Yao(2009)]. We note that even if similar mathematical frameworks underlie these approaches, they have different semantics and usage. Such three-valued sets lead to three-way decision processes $[\mathrm{Yao}(2012)]$, that leave room for a non-committal attitude that differs from the mere acceptance and rejection of options.

One may argue that since Boolean logic is so basic for all kinds of scientific investigations, three-valued logic may be useful and as basic for a number of useful reasoning tasks. However there is no clear consensus about this claim, and even there is some confusion as to the potential uses of a third truth value, different from true and false. Indeed, there are various ways of interpreting it. We can comment on some of them:

- Possible: this interpretation was proposed by Lukasiewicz [Borowski(1970)], the pioneer of multivalued logic. He motivated his departure from bivalence by the wish do deal with future contingents in a truth-functional way. A proposition is Possible if its truth-value will be only known in the future. Note that this question is better handled in temporal modal logics, nowadays, that is, Possible is more a modality than a truth-value.

- Unknown: this interpretation is proposed by Kleene [Kleene(1952)] in 1952, and it is in some sense closely related to the previous one. A proposition is unknown if its (Boolean) truth-value cannot be computed for some reason (for instance, it is too time-consuming to do it). This interpretation has been very popular in various areas of computer science nowadays, and may lead to paradoxes, if truth-functionality is enforced [Urquhart(1986), Dubois and Prade(2001)]. This view of the third truthvalue also pervades the intuitions behind interval sets [Yao(2009)] and rough sets [Ciucci and Dubois(2010)]. It also suffers a limited expressive power: it can only account for epistemic states in terms of partial models, assigning an epistemic status to atoms only [Dubois(2008)].

- Undefined: this is another proposal by Kleene, which applies to Boolean formulas viewed as recursive functions. The undefined state corresponds to the choice of the argument of the function outside its definition domain. This kind of truth value usually contaminates the result of the computation of the recursive function, that is the truth-value of a proposition involving undefined atoms is Undefined.

- Half-true: This is typical of fuzzy logic [Hájek(1998)]. The intuition is that for some propositions, truth is a matter of degree. So the extension of the corresponding predicate involves elements that are peripheral, or borderline, as opposed to elements that are central. This is very natural for representing some words in natural language like bird, tall, etc. A robin is a prototypical bird (a central element) while a penguin is untypical as a bird (a peripheral element). The use of three-valued sets in linguistics goes back to Gentilhomme [Gentilhomme(1968)]. In the case of words such as tall, the continuous representation of the height scale pleads in favor of a truth set larger than $\mathbf{3}$, namely the unit interval for instance, as commonly used in fuzzy logic. Shadowed sets [Pedrycz(2009)] are based on the idea 
of turning fuzzy sets into three-valued sets using two cuts generalizing the core and support of a fuzzy set.

- Irrelevant: the idea is that in some possible worlds, atomic propositions are not applicable. For instance, the proposition "My car is blue" is neither true nor false if I have no car. However, there is no contamination effect in this case, namely if a component of a proposition is irrelevant, it is supposed not to take part in the evaluation of the truth of the whole. An example of such a three-valued entity is a rule "if $A$ then $B$ ", viewed as a conditional event $B \mid A$ [Dubois and Prade(1994)], $A$ and $B$ being Boolean propositions. When the antecedent $A$ is false, the rule $B \mid A$ is irrelevant as it cannot be applied. So it has no role in the computation of a logical combination with other rules.

- Inconsistent: the third value stands for a proposition which is both true and false, so, in some sense, it is the dual of "unknown". Several works try to tame the notion of contradiction by means of a truth value (for instance, Priest [Priest(1979)] and Belnap [Belnap(1977)]). The stance of some paraconsistent logics is to treat contradictions locally without losing inference capabilities (for instance [Asenjo and Tamburino(1975)]). The possible confusion between usual truth-values and the notion of contradiction has been criticized as also generating paradoxes [Fox(1990), Dubois(2008)].

The possible meanings of the third truth-value mentioned above are probably not exhaustive, but they are enough to lay bare an opposition between two kinds of understanding of the third truth value, we could call ontological and epistemic, respectively. The third truth-value is sometimes intrinsic to the definition of propositions: Undefined is needed for defining partial functions, Half-true for defining gradual or fuzzy predicates, Irrelevant is intrinsic to the commonsense notion of a rule that does not apply to some situations. Such truth-values are ontological, that is, satisfiability is defined in terms of interpretations $w \vDash p$ having three values [Lawry and Tang(2012)]. On the other hand, Unknown and Possible refer to situations where the Boolean nature of propositions is not questioned but their truth or falsity has not been yet established, but can eventually be so, by waiting for the proper moment, or by means of a stronger computer, or by bringing the proper information that is missing. Such truth-values are epistemic. They refer to the state of knowledge of an agent relative to propositions that are ontologically Boolean. In this case, the third truth-value refers not to the proposition it is supposed to qualify, but to the state of information concerning this proposition. These considerations drive us close to epistemic logics. The relationships between three-valued logics and epistemic logics are studied in [Ciucci and Dubois(2012b)]. Here, we focus on the relationships among three-valued logics.

Clearly, Boolean connectives can be extended to three-valued logics in various ways, but there is only a finite number of logical 3-valued functions of a given arity. Contrary to the Boolean case, where the basic connectives, say conjunction and negation, are uniquely defined, there are degrees of freedom in 3-valued logics for the definition of such connectives. The choice of connectives can be driven by the algebraic properties one may wish to salvage, given that the Boolean structure is no longer possible. However this choice should also be driven by the preservation of Boolean tables for the two extreme truth-values and by the meaning of the third one. For instance, if it means half-true one may argue that the conjunction of 
a true proposition with an half-true one cannot be more than half-true in turn. In contrast if the third truth value means irrelevant, it looks plausible that the conjunction of a true proposition with an irrelevant one should remain true, as the second proposition is viewed as counting for nothing.

In the following, we extend and complete our study [Ciucci and Dubois(2012a)] on three-valued connectives. First, we use a definition of conjunction, implication and negation obeying minimal intuitively meaningful properties and show that there are only 14 different such conjunctions and implications, and 3 negations, on threevalued sets. We also discuss the relationships between other definitions of conjunctions in many-valued logics, such as t-norms and uninorms [Klement et al.(2000)Klement, Mesiar, and Pap]. Further, generalising the connections between implication, entailment and conjunction in classical logic, we study the relationships among all these operations via standard transformations involving or not an involutive negation [Dubois and Prade(1984a), Fodor(1991)] and classify them according to their mutual behaviour. It turns out that all the connectives can be mutually defined, so that starting from a simple structure we can obtain all of them. Finally, we show that any binary connective is definable using Łukasiewicz logic connectives, provided that the third truth value is part of the language.

\section{Connectives on Three Values}

A three-valued set on the universe $X$ can be defined as a mapping $f: X \mapsto \mathbf{3}$, where $\mathbf{3}=(\{F, N, T\},>)$ is a chain of truth values. Namely, the set $\{F, N, T\}$ is equipped with the ordering relation $T>N>F$, often called the truth-ordering [Belnap(1977)], with $T$ meaning true, $F$ meaning false and $N$ (for neutral) is the third truth-value standing in between. We denote by $\vee$ and $\wedge$ the maximum and minimum operations on this set.

The structure $(\mathbf{3},>)$ is the simplest bipolar scale, namely a scale where positive and negative areas are separated by a neutral value. Given the importance of bipolarity in human cognition [Dubois and Prade(2008)], one may suspect that some three-valued logics should play in knowledge representation a role similar to Boolean logics for mathematical reasoning. However, there is no consensus along this line so far, partly due to the multiplicity of possible three-valued systems. This is one of the motivations of this paper, namely, study relationships between three-valued functions, and proceed toward isolating some "useful" three-valued logics.

2.1. Conjunctions. First we give a general definition of a conjunction on $\mathbf{3}$. At the end of the section, other possible definitions are discussed.

Definition 1. A conjunction on $\mathbf{3}$ is a binary mapping $*: \mathbf{3} \times \mathbf{3} \mapsto \mathbf{3}$ such that

(C1) If $x \leq y$ then $x * z \leq y * z$ (left monotonicity);

(C2) If $x \leq y$ then $z * x \leq z * y$ (right monotonicity);

(C3) $F * F=F * T=T * F=F$ and $T * T=T$ (conformity with Boolean logic).

This is clearly a minimal definition. Note that $N * F=F * N=F$ in all cases since $N \leq T$ and due to (C1) and (C3) we get $N * F \leq T * F=F$, and similarly $F * N \leq F * T=F$ from (C2) and (C3). Now, by listing all the possible conjunctions on 3 we get 14 possibilities, according to Table 1 .

This framework, even if encompassing, is not able to cover some identified conjunction connectives, for instance, Kleene's weak conjunction for undefined (also 
TABLE 1. All conjunctions on $\mathbf{3}$ according to Definition 1

\begin{tabular}{c|c|c|c}
$*$ & $\mathrm{~F}$ & $\mathrm{~N}$ & $\mathrm{~T}$ \\
\hline $\mathrm{F}$ & $\mathrm{F}$ & $\mathrm{F}$ & $\mathrm{F}$ \\
\hline $\mathrm{N}$ & $\mathrm{F}$ & & \\
\hline $\mathrm{T}$ & $\mathrm{F}$ & & $\mathrm{T}$
\end{tabular}

\begin{tabular}{c|c|c|c|c|c|c|c} 
n. & $N * N$ & $T * N$ & $N * T$ & $\mathrm{C} 4$ & $\mathrm{C} 5$ & $\mathrm{C} 6$ & \\
\hline \hline 1 & $\mathrm{~T}$ & $\mathrm{~T}$ & $\mathrm{~T}$ & yes & yes & no & Sette \\
\hline \hline 2 & $\mathrm{~N}$ & $\mathrm{~T}$ & $\mathrm{~T}$ & yes & yes & no & quasi conjunction/Sobociński \\
\hline 3 & $\mathrm{~N}$ & $\mathrm{~T}$ & $\mathrm{~N}$ & no & yes & no & \\
\hline 4 & $\mathrm{~N}$ & $\mathrm{~N}$ & $\mathrm{~T}$ & no & yes & no & \\
\hline 5 & $\mathrm{~N}$ & $\mathrm{~N}$ & $\mathrm{~N}$ & yes & yes & yes & min/interval conjunction/Kleene \\
\hline \hline 6 & $\mathrm{~F}$ & $\mathrm{~F}$ & $\mathrm{~T}$ & no & no & no & \\
\hline 7 & $\mathrm{~F}$ & $\mathrm{~F}$ & $\mathrm{~N}$ & no & yes & no & \\
\hline 8 & $\mathrm{~F}$ & $\mathrm{~F}$ & $\mathrm{~F}$ & yes & yes & no & Bochvar external \\
\hline 9 & $\mathrm{~F}$ & $\mathrm{~N}$ & $\mathrm{~F}$ & no & yes & no & \\
\hline 10 & $\mathrm{~F}$ & $\mathrm{~N}$ & $\mathrm{~T}$ & no & no & no & \\
\hline 11 & $\mathrm{~F}$ & $\mathrm{~N}$ & $\mathrm{~N}$ & yes & yes & yes & Eukasiewicz \\
\hline 12 & $\mathrm{~F}$ & $\mathrm{~T}$ & $\mathrm{~F}$ & no & no & no & \\
\hline 13 & $\mathrm{~F}$ & $\mathrm{~T}$ & $\mathrm{~N}$ & no & no & no & \\
\hline 14 & $\mathrm{~F}$ & $\mathrm{~T}$ & $\mathrm{~T}$ & yes & no & no &
\end{tabular}

known as Bochvar internal conjunction) [Kleene(1952), Bochvar(1981)] and McCarthy logic conjunction [McCarthy(1963), Konikowska(1996)]. These conjunctions are indeed not monotonic, as $N * F=N$.

Other important usual properties for conjunction, not imperatively requested here, are commutativity, associativity and the identity role played by $T$ :

(C4) $x * y=y * x$ (commutativity);

(C5) $x *(y * z)=(x * y) * x$ (associativity);

(C6) $T * x=x$ and $x * T=x$ (identity).

As can be seen, only the conjunctions $1,2,5,8,11,14$ are commutative. Noncommutative conjunctions can be paired by noticing they are mutually definable by swapping $T$ and $N$, i.e., if $T * N=a$ and $N * T=b$ then $T \star N=b$ and $N \star T=a$ for another conjunction $\star$. These pairs of conjunctions are $(3,4) ;(6,12) ;(7,9)$ and $(10,13)$. When symmetrizing these conjunctions we get the following result.

Proposition 1. Let $*^{\prime}, *^{\prime \prime}: \mathbf{3} \mapsto \mathbf{3}$ be defined as $a *^{\prime} b:=b *^{\prime} a=(a * b) \wedge(b * a)$ and $a *^{\prime \prime} b=b *^{\prime \prime} a=(a * b) \vee(b * a)$. Then, we have:

- $*_{3}^{\prime}=*_{4}^{\prime}=*_{5}(\mathrm{~min}), *^{\prime \prime}{ }_{3}=*^{\prime \prime}{ }_{4}=*_{2}$ (Sobocinski);

- $*_{6}^{\prime}=*_{7}^{\prime}=*_{9}^{\prime}=*_{12}^{\prime}=*_{8}$ (Bochvar), $*^{\prime \prime}{ }_{7}=*^{\prime \prime}{ }_{9}=*_{11}$ (Eukasiewicz);

- $*_{10}^{\prime}=*_{13}^{\prime}=*{ }_{11}$ (Eukasiewicz), $*^{\prime \prime}{ }_{6}=*^{\prime \prime}{ }_{10}=*^{\prime \prime}{ }_{12}=*^{\prime \prime}{ }_{13}=*{ }_{14}$.

Proof. A straightforward application of $*^{\prime}, *^{\prime \prime}$ definition to Table 1 , where it is enough to consider the columns $T * N, N * T$ and take respectively the min and the $\max$. 
Moreover only Lukasiewicz and Kleene conjunctions have identity $T$. They are associative conjunctions, a property satisfied by the majority of the 14 connectives. Łukasiewicz and Kleene conjunctions are the only two t-norms [Klement et al.(2000)Klement, Mesiar, and Pap] remaining (in this setting, Łukasiewicz t-norm is the least t-norm as well). Interestingly, Sobociński conjunction has identity $N$, and is the only one to be so, which makes it a good candidate for modeling irrelevance, and is also associative.

In conclusion, from the original 14 conjunctions, we get only 6 which are commutative. If we restrict our choice further to the associative ones we get 5 solutions, which are the only ones with names, that is to say, the only ones in the table (to the best of our knowledge) that have been studied in literature.

\section{Remark 1. Other definitions of conjunction.}

There have been several other attempts to define generalized conjunctions in the three-valued setting, that we review now.

Conjunctions of conditional events. In [Walker(1994)], Walker discussed how to extend Boolean conjunctions to conditional events of the form $A \mid B$ representing rules of the form if $B$ then $A$. They can be represented by orthopairs of elements of a Boolean algebra (sets with empty intersection), respectively corresponding to $A \cap$ $B$ (examples of the rule) and $A^{c} \cap B$ (counterexamples), where $A^{c}$ is the complement of set $A$. The conditions he imposes on these conjunctions are: coincidence with Boolean conjunction on Boolean values $\{0,1\}$, idempotence and commutativity (we note that the same requirements are also used in [Adamatzky(2003)]).

Then, due to the bijection between conditional events and three-valued sets, it is possible to interpret the operations as three-valued truth tables. As a result, there are only nine possibilities, among which we can find weak Kleene, (strong) Kleene and Sobociński conjunctions. Sobociński's conjunction has been used to define a non-monotonic logic of conditionals with three-valued semantics [Dubois and Prade(1994)].

The other six conjunctions are given in Table 2. We note that Walker also defines disjunction by De Morgan properties with respect to the standard involutive negation.

TABLE 2. Walker conjunctions (the original numbering is given)

\begin{tabular}{c|c|c|c}
$*_{W}^{2}$ & $\mathrm{~F}$ & $\mathrm{~N}$ & $\mathrm{~T}$ \\
\hline $\mathrm{F}$ & $\mathrm{F}$ & $\mathrm{N}$ & $\mathrm{F}$ \\
\hline $\mathrm{N}$ & $\mathrm{N}$ & $\mathrm{N}$ & $\mathrm{F}$ \\
\hline $\mathrm{T}$ & $\mathrm{F}$ & $\mathrm{F}$ & $\mathrm{T}$ \\
$*_{W}^{6}$ & $\mathrm{~F}$ & $\mathrm{~N}$ & $\mathrm{~T}$ \\
\hline $\mathrm{F}$ & $\mathrm{F}$ & $\mathrm{T}$ & $\mathrm{F}$ \\
\hline $\mathrm{N}$ & $\mathrm{T}$ & $\mathrm{N}$ & $\mathrm{N}$ \\
\hline $\mathrm{T}$ & $\mathrm{F}$ & $\mathrm{N}$ & $\mathrm{T}$
\end{tabular}

\begin{tabular}{c|c|c|c}
$*_{W}^{4}$ & $\mathrm{~F}$ & $\mathrm{~N}$ & $\mathrm{~T}$ \\
\hline $\mathrm{F}$ & $\mathrm{F}$ & $\mathrm{F}$ & $\mathrm{F}$ \\
\hline $\mathrm{N}$ & $\mathrm{F}$ & $\mathrm{N}$ & $\mathrm{F}$ \\
\hline $\mathrm{T}$ & $\mathrm{F}$ & $\mathrm{F}$ & $\mathrm{T}$ \\
$*_{W}^{7}$ & $\mathrm{~F}$ & $\mathrm{~N}$ & $\mathrm{~T}$ \\
\hline $\mathrm{F}$ & $\mathrm{F}$ & $\mathrm{T}$ & $\mathrm{F}$ \\
\hline $\mathrm{N}$ & $\mathrm{T}$ & $\mathrm{N}$ & $\mathrm{T}$ \\
\hline $\mathrm{T}$ & $\mathrm{F}$ & $\mathrm{T}$ & $\mathrm{T}$
\end{tabular}

\begin{tabular}{c|c|c|c}
$*_{W}^{5}$ & $\mathrm{~F}$ & $\mathrm{~N}$ & $\mathrm{~T}$ \\
\hline $\mathrm{F}$ & $\mathrm{F}$ & $\mathrm{N}$ & $\mathrm{F}$ \\
\hline $\mathrm{N}$ & $\mathrm{N}$ & $\mathrm{N}$ & $\mathrm{T}$ \\
\hline $\mathrm{T}$ & $\mathrm{F}$ & $\mathrm{T}$ & $\mathrm{T}$ \\
$*_{W}^{8}$ & $\mathrm{~F}$ & $\mathrm{~N}$ & $\mathrm{~T}$ \\
\hline $\mathrm{F}$ & $\mathrm{F}$ & $\mathrm{T}$ & $\mathrm{F}$ \\
\hline $\mathrm{N}$ & $\mathrm{T}$ & $\mathrm{N}$ & $\mathrm{F}$ \\
\hline $\mathrm{T}$ & $\mathrm{F}$ & $\mathrm{F}$ & $\mathrm{T}$
\end{tabular}

Among these conjunctions, the only associative one is $*_{W}^{4}$ and they are all nonmonotone. This behavior, as well as the fact that $N *_{W}^{i} F=T$ for $i=6,7,8$, casts some doubts about their interpretability.

Uninorms. The idea is to generalize the notion of t-norm and t-conorm by letting any element $e$ (usually, in the unit interval) be the identity. 
Definition 2. [Mas et al.(1999b)Mas, Mayor, and Torrens] A uninorm is a binary operator which is associative, commutative, non decreasing in each component and with identity $e: \forall x, e * x=x$.

In the three-valued setting, we do not obtain any new connective, all the definable uninorms also satisfy Definition 1.

Proposition 2. The only 6 uninorms definable on $\mathbf{3}$ are: Lukasiewicz, Kleene and Sobociński conjunctions and disjunction.

Proof. We can have three choices for the neutral element:

- the neutral element is $e=T$. Then we get a t-norm, which on three values comes down to Lukasiewicz and Kleene conjunctions;

- if $e=N$, we get Sobociński conjunction and disjunction (operation 2 in Ta-

ble 1). It is the only conjunctive discrete uninorm [De Baets et al.(2009)De Baets, Fodor, Ruiz-Aguilera, and the most elementary such connective (as there are no uninorms in the Boolean setting);

- if $e=F$ we have a t-conorm, which on three values corresponds to Eukasiewicz and Kleene disjunction.

T-operators. This is another generalization of t-norms on pre-ordered sets [Mas et al.(1999a)Mas, Mayor, and Tor

Definition 3. [Mas et al.(1999b)Mas, Mayor, and Torrens] A binary operator * on a finite scale $\left\{F<x_{1}<\ldots<T\right\}$ is named t-operator if it is associative, commutative, such that $F * F=F, T * T=T$ and it satisfies 1-smoothness: $x_{i} *$ $x_{j-1} \leq x_{i} * x_{j}$ and if $x_{i} * x_{j}=x_{k}$ then $x_{k-1} \leq x_{i} * x_{j}$.

Clearly, the following property holds.

Lemma 3. Definition 3 implies monotonicity conditions (C1) and (C2) in Definition 1 .

Proof. Let us suppose that $x_{i} \leq x_{j}$ then, we can find a chain $x_{i} \leq x_{i+1} \cdots \leq$ $x_{j-1} \leq x_{j}$. Thus applying 1 -smoothness and commutativity we obtain (C1) and $(\mathrm{C} 2)$.

Proposition 4. On three-valued scales, there are only five t-operators: Kleene and Lukasiewicz conjunctions and disjunctions and the aggregation operator in Table 3.

Proof. This result can be obtained by Lemma 3, requiring also condition if $x_{i} * x_{j}=$ $x_{k}$ then $x_{k-1} \leq x_{i} * x_{j}$ on the associative and commutative operations obtained in Table 1. Then, we see that the only operator satisfying Definition 3 and such that $T * F=F * T \neq F$ is the median operator.

TABLE 3. The median t-operator

\begin{tabular}{c|c|c|c}
$*_{t}$ & $\mathrm{~F}$ & $\mathrm{~N}$ & $\mathrm{~T}$ \\
\hline $\mathrm{F}$ & $\mathrm{F}$ & $\mathrm{N}$ & $\mathrm{N}$ \\
\hline $\mathrm{N}$ & $\mathrm{N}$ & $\mathrm{N}$ & $\mathrm{N}$ \\
\hline $\mathrm{T}$ & $\mathrm{N}$ & $\mathrm{N}$ & $\mathrm{T}$
\end{tabular}


The peculiarity on the operator in Table 3 is that it does not generalise Boolean connectives: $F$ and $T$ yield the third value $N$. In fact it is easy to see that it is the operation med $(x, y, N)$ computing the median between $x, y$ and $N$. It is known to be the only associative operation between $\wedge$ and $\vee$, and a special case of Sugeno Integral.

2.2. Implications. We turn now our attention to the implication connective, starting again from a general definition, with requirements on monotonicity and coincidence with classical logic on $\{F, T\}$.

Definition 4. An implication on $\mathbf{3}$ is a binary mapping $\rightarrow$ from $\mathbf{3} \times \mathbf{3}$ to $\mathbf{3}$ such that

(I1) If $x \leq y$ then $y \rightarrow z \leq x \rightarrow z$;

(I2) If $x \leq y$ then $z \rightarrow x \leq z \rightarrow y$;

(I3) $F \rightarrow F=T \rightarrow T=T$ and $T \rightarrow F=F$.

From the above definition we have

Proposition 5. From (I1)-(I3) it follows that for all $x, x \rightarrow T=T$ and, in particular, $F \rightarrow T=T$.

Proof. From (I1) and $y=z=T$ we have $x \leq T$ implies $T \rightarrow T \leq x \rightarrow T$ and by (I3) we get the thesis.

Thus, this definition extends in a coherent manner the usual definition of material implication on $\{F, T\}$. This definition can be found, for instance in [Sabo(1998), Mas et al.(2007)Mas, Monserrat, Torrens, and Trillias] and is quite general. Nevertheless, as discussed in [Mas et al.(2007)Mas, Monserrat, Torrens, and Trillias] it does not cover all existing implications, for instance, the weak Kleene implication [Kleene(1952)]. Now, we list all definable implications according to Definition 4. It yields the (incomplete) truth table in Table 4 on the top and the further monotonicity constraint $N \rightarrow N \geq\{T \rightarrow N, N \rightarrow F\}$. Thus, the three missing values can be filled also in this case in 14 different ways.

Additional interesting properties worth considering, are

(I4) $T \rightarrow x=x$ (left neutrality or boundary condition);

(I5) $x \rightarrow y=T$ iff $x \leq y$ (ordering property).

We remark that (I5) is considered by some authors [Hardegree(1981), Smets and Magrez(1987)] as a fundamental condition for implication. Table 4 summarizes all possible implications in agreement with our definition, showing also the only two ones that satisfy additional conditions I4 and I5. The latter correspond to the restriction of infinitevalued Łukasiewicz and Gödel implications, respectively defined for $x, y \in[0,1]$ as:

$$
\begin{aligned}
& x \rightarrow_{L} y:=\min \{1,1-x+y\} \\
& x \rightarrow_{G} y:= \begin{cases}1 & x \leq y \\
y & x>y .\end{cases}
\end{aligned}
$$

to the present three-valued setting. They are the t-norms residua [Klement et al.(2000)Klement, Mesiar, and Pap Mas et al.(2004)Mas, Monserrat, and Torrens] of Łukasiewicz and Gödel conjunctions. 
TABle 4. All implications according to Definition 4

\begin{tabular}{c|c|c|c}
$\rightarrow$ & $\mathrm{F}$ & $\mathrm{N}$ & $\mathrm{T}$ \\
\hline $\mathrm{F}$ & $\mathrm{T}$ & $\mathrm{T}$ & $\mathrm{T}$ \\
\hline $\mathrm{N}$ & & & $\mathrm{T}$ \\
\hline $\mathrm{T}$ & $\mathrm{F}$ & & $\mathrm{T}$
\end{tabular}

\begin{tabular}{c|c|c|c|c|c|c}
$\mathrm{n}$. & $\mathrm{N} \rightarrow \mathrm{N}$ & $\mathrm{T} \rightarrow \mathrm{N}$ & $\mathrm{N} \rightarrow \mathrm{F}$ & $\mathrm{I} 4$ & $\mathrm{I} 5$ & \\
\hline \hline 1 & $\mathrm{~F}$ & $\mathrm{~F}$ & $\mathrm{~F}$ & no & no & \\
\hline \hline 2 & $\mathrm{~N}$ & $\mathrm{~F}$ & $\mathrm{~F}$ & no & no & Sobociński \\
\hline 3 & $\mathrm{~N}$ & $\mathrm{~F}$ & $\mathrm{~N}$ & no & no & \\
\hline 4 & $\mathrm{~N}$ & $\mathrm{~N}$ & $\mathrm{~F}$ & yes & no & Jaśkowski \\
\hline 5 & $\mathrm{~N}$ & $\mathrm{~N}$ & $\mathrm{~N}$ & yes & no & (strong) Kleene \\
\hline \hline 6 & $\mathrm{~T}$ & $\mathrm{~T}$ & $\mathrm{~F}$ & no & no & Sette \\
\hline 7 & $\mathrm{~T}$ & $\mathrm{~T}$ & $\mathrm{~N}$ & no & no & \\
\hline 8 & $\mathrm{~T}$ & $\mathrm{~T}$ & $\mathrm{~T}$ & no & no & \\
\hline 9 & $\mathrm{~T}$ & $\mathrm{~N}$ & $\mathrm{~T}$ & yes & no & Nelson \\
\hline 10 & $\mathrm{~T}$ & $\mathrm{~N}$ & $\mathrm{~F}$ & yes & yes & Gödel \\
\hline 11 & $\mathrm{~T}$ & $\mathrm{~N}$ & $\mathrm{~N}$ & yes & yes & Łukasiewicz \\
\hline 12 & $\mathrm{~T}$ & $\mathrm{~F}$ & $\mathrm{~T}$ & no & no & Bochvar external \\
\hline 13 & $\mathrm{~T}$ & $\mathrm{~F}$ & $\mathrm{~N}$ & no & yes & \\
\hline 14 & $\mathrm{~T}$ & $\mathrm{~F}$ & $\mathrm{~F}$ & no & yes & Gaines-Rescher
\end{tabular}

Remark 2. The algebraic structure defined in order to treat at the same time Łukasiewicz and Gödel logic is known as Heyting Wajsberg (HW) algebra [Cattaneo et al.(2004a)Cattaneo, Ciucc We also note that HW algebras are equivalent to other well known structures, without entering into details (see [Cattaneo et al.(2004b)Cattaneo, Ciucci, Giuntini, and Konig]) we mention:

- $\mathrm{MV}_{\Delta}$ algebras, obtained adding a Baaz's operator $\Delta$ [Baaz(1996), Hájek(1998)] to MV algebras;

- Stonean MV algebra, a particular class of MV algebras introduced by Belluce [Belluce(1997)].

- $\mathrm{BZMV}^{d M}$ algebras, a pasting of MV algebras and BZ lattices [Cattaneo et al.(1999)Cattaneo, Giuntini, a

On Table 4, we can see that among the 14 implications we also have the Kleene, the Gaines-Rescher, the Nelson [Vakarelov(1977), Cignoli(1986)], the Sobociński [Sobociński(1952)], the Jaśkowski ${ }^{4}$ [Jaśkowski(1969)] and the Sette [Sette(1973)] ones.

Remark 3. In his study on invariant fuzzy implications [Drewniak(2006)], Drewniak points out 18 implications which are minimal "invariant with respect to the family of bijections". Once considered on three values, they reduce only to nine implications, which corresponds to implications 1,2,4,6,8,9,10,12,14 in Table 4 .

2.3. Negations. From the above implications we can derive in a standard way a negation as $x^{\prime}:=x \rightarrow F$. Of course we can obtain only three different negations depending on the value assigned to $N^{\prime}$. Indeed, the negation of the Boolean values

\footnotetext{
${ }^{4}$ This implication is ascribed to Słupecki by Jaśkowski in [Jaśkowski(1969)].
} 
is constrained by $F^{\prime}=T$ and $T^{\prime}=F$. Thus, we can cluster the 14 implications in three groups, according to the negation they produce:

- $\sim N=F$, that is we obtain an intuitionistic negation. Implications $1,2,4$, $6,10,14$ belong to this group.

- $\neg N=N$. In this case, the negation is involutive. The group includes implications 3, 5, 7, 11, 13.

- $-N=T$. This negation corresponds to a paraconsistent one. All remaining implications belong to this cluster, namely, implications 8, 9, 12 .

In Table 4, it is interesting to distinguish between implications that obey the contraposition law with respect to the involutive negation and those which do not. Namely implications I1, I2 (Sobociński), I5, I8, I11 (Łukasiewicz), and I14 (GainesRescher) are their own contraposed. Others go by pair made of an implication and its contraposed, namely (I3, I4) (I6, I12), (I7, I9) (I10, I13). The involutive negation enables 14 disjunctions + to be generated from the 14 conjunctions $*$ as $a+b=\neg(\neg a * \neg b)$. Using implications, disjunctions can also be obtained in the form $a+b=\neg a \rightarrow b$.

\section{From Conjunction to Implication and Back}

In [Dubois and Prade(1984a), Dubois and Prade(1984b)] a generation process for fuzzy set connectives is introduced. It enables relationships among implications and conjunctions to be laid bare. We now apply this methodology to the three truth values case.

Given a binary operation $\odot$ on $\{F, N, T\}$ and the standard involutive negation $\neg F=T, \neg N=N, \neg T=F$, we introduce the following transformations of $\odot$ :

$$
\begin{aligned}
& a[\mathcal{E}(\odot)] b=b \odot a \\
& \text { (exchange) } \\
& a[\mathcal{C}(\odot)] b=\neg b \odot \neg a \\
& \text { (contraposition) } \\
& \text { (1c) } \quad a[\mathcal{S}(\odot)] b=\neg(a \odot \neg b) \\
& \text { (material implication) } \\
& a[\mathcal{R}(\odot)] b= \begin{cases}0 & \nexists s, a \odot s \leq b \\
\sup \{s: a \odot s \leq b\} & \text { otherwise }\end{cases}
\end{aligned}
$$

They can be used to generate one operation from another. In particular, from a non-commutative conjunction we can obtain another conjunction with the exchange transformation $\mathcal{E}$. From a conjunction, we can also respectively obtain two implications by residuation $\mathcal{R}$ and transformation $\mathcal{S}$ based on involutive negation only. The latter comes down to mimicking the definition of material implication. Conversely, starting from an implication we can obtain another implication using contraposition $\mathcal{C}$ and a conjunction by residuation $\mathcal{R}$ applied to an implication. Transformation $\mathcal{S}$ is involutive, as $a[\mathcal{S}[\mathcal{S}(\odot)]] b=a \odot b$.

The interesting thing is that the application of $\mathcal{E}, \mathcal{C}$ and $\mathcal{S}$ to the above discussed 28 connectives does not generate any new connective. The same remark holds to a lesser extent for $\mathcal{R}$. Below we present relationships between implications and conjunctions induced from these transformations, clustered into four different classes corresponding to different patterns.

3.1. Class 1: self-definable conjunctions-implications pairs : 1, 2 and 11. In this first pattern, implication and conjunction are mutually definable like in 
classical logic and no other operation is obtainable by the transformations.
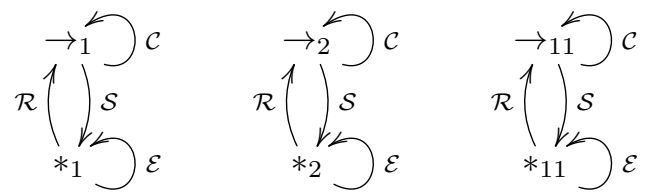

With no surprise, in this group we find Lukasiewicz implication and conjunction (n.11). A formal axiom system for $\left(\rightarrow_{11}, *_{11},+_{11}, \neg\right)$ is well-known, where $+_{11}$ is the bounded sum. Also, Sobociński logic [Sobociński(1952)] possesses an axiomatization based on $\left(\rightarrow_{2}, *_{2},+_{2}, \neg\right)$ where $+_{2}$ can be defined as $x+{ }_{2} y:=\neg x \rightarrow_{2} y$, and the designated values are both $\mathrm{T}$ and $\mathrm{N}$. Here, the interpretation given to the third value is irrelevant: moreover, the (conjunctive) discrete uninorm $*_{2}$ has implication $\rightarrow_{2}$ for its residuum [Baets and Fodor(1999), Mas et al.(1999b)Mas, Mayor, and Torrens] (as also discussed later). We also note that Sette conjunction (n.1) corresponds to the collapse of the truth values $\mathrm{T}$ and N. This also happens with Sette implication (n.6) which however appears in the schema of class 3. So the closure of these sets of connectives for these transformations is not surprising.

Remark 4. We note that Sette logic has been obtained in [D'Ottaviano and da Costa(1970)] from Łukasiewicz logic with a view to demonstrate a relationship between manyvalued logic and paraconsistent logic. Given Łukasiewicz three-valued logic (with two designated values), Sette operations are obtained as follows:

$$
\begin{aligned}
p \wedge_{S e} q & :=\neg(p \wedge q) \rightarrow_{L}(p \wedge q) \\
p \rightarrow_{S e} q & :=J(q) \rightarrow_{L} J(p)
\end{aligned}
$$

where $J(p)=\neg p \wedge_{S e} \neg\left(p \wedge_{S e} \neg p\right)$ and $\wedge$ denotes the minimum.

3.2. Class 2: implications $\{\mathbf{3}, \mathbf{4}, \mathbf{1 4}\}$ and $\{\mathbf{5}, \mathbf{1 0}, \mathbf{1 3}\}$. Also this pattern is closed, but it involves three implications and three conjunctions.
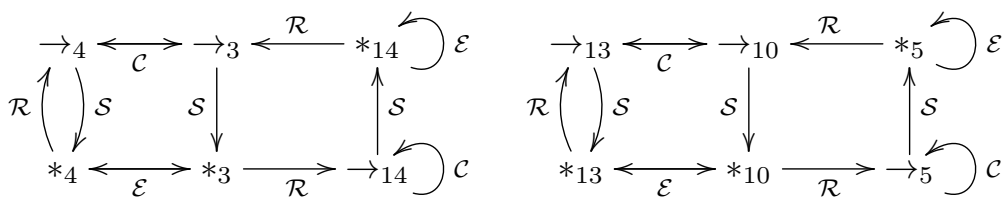

Let us note that while most implications in these groups are well known (Jaśkowski (n.4), Gaines-Rescher (n.14), Kleene (n.5) and Gödel (n.10)), only one conjunction (minimum, n.5) has focused attention in the literature. Indeed, when these implications are put into a formal logical system, the considered conjunction is usually the minimum. More precisely, we have three-valued Gödel logic $\left(\rightarrow_{10}, *_{5}, \vee, \sim\right)$ which is the well-known intuitionistic (Heyting) logic plus the prelinearity axiom $(x \rightarrow$ $y) \vee(y \rightarrow x)$ and the Hosoi condition to obtain three values [Hosoi(1996)], or equivalently BL logic plus the idempotency of the conjunction $(a * a=a)$ [Hájek(1998)] and again the three-valuedness condition. We note that this logic is also known in the Answer Set Programming field as the here-and-there logic [Pearce(2006)]. The logical system derived by Jaśkowski implication $\left(\rightarrow_{4}, *_{5}, \vee, \neg\right)$ has been studied by several authors in the field of paraconsistent logic [D'Ottaviano and da Costa(1970), Asenjo and Tamburino(1975), Avron(1986)], where the designated values are $N$ and $T$. It can be proved equivalent to Sobociński logic (n.2) through the following 
identities:

$$
\begin{aligned}
& p \rightarrow_{S} q=\left(p \rightarrow_{J} q\right) \wedge\left(\neg q \rightarrow_{J} \neg p\right) \\
& p \rightarrow_{J} q=q \vee\left(p \rightarrow_{S} q\right)
\end{aligned}
$$

3.3. Class 3: implications $\{\mathbf{6 , 1 2}\}$ and $\{\mathbf{7 , 9}\}$. This pattern is not closed, that is $\mathcal{R}\left(*_{6}\right)$ and $\mathcal{R}\left(*_{7}\right)$ yield operations that do not belong to the 14 implications presented in the previous sections.
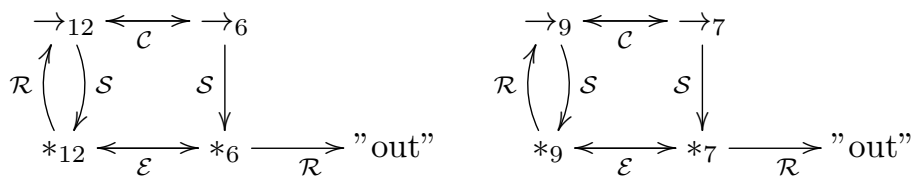

In the above patterns, the value "out" means that the implication operator $\mathcal{R}(*)$ does not satisfy all the constraints of Definition 4 . Full truth tables of these external implications are given in Table 5.

TABLE 5. Residuum of conjunctions $*_{6}$ and $*_{7}$.

\begin{tabular}{c|c|c|c}
$\mathcal{R}\left(*_{6}\right)$ & $\mathrm{F}$ & $\mathrm{N}$ & $\mathrm{T}$ \\
\hline $\mathrm{F}$ & $\mathrm{T}$ & $\mathrm{T}$ & $\mathrm{T}$ \\
\hline $\mathrm{N}$ & $\mathrm{N}$ & $\mathrm{N}$ & $\mathrm{T}$ \\
\hline $\mathrm{T}$ & $\mathrm{N}$ & $\mathrm{N}$ & $\mathrm{T}$
\end{tabular}

\begin{tabular}{c|c|c|c}
$\mathcal{R}\left(*_{7}\right)$ & $\mathrm{F}$ & $\mathrm{N}$ & $\mathrm{T}$ \\
\hline $\mathrm{F}$ & $\mathrm{T}$ & $\mathrm{T}$ & $\mathrm{T}$ \\
\hline $\mathrm{N}$ & $\mathrm{N}$ & $\mathrm{T}$ & $\mathrm{T}$ \\
\hline $\mathrm{T}$ & $\mathrm{N}$ & $\mathrm{N}$ & $\mathrm{T}$
\end{tabular}

As can be seen, the problem lies in the value of $T \rightarrow_{\mathcal{R}\left(*_{x}\right)} F$ which in cases $x=6,7,8$ is $N$ instead of $F$. These new connectives do not extend classical logic. This is a consequence of a more general result which applies to all many-valued operators, not just to the three-valued set $\mathbf{3}$, as proved in the following proposition.

Proposition 6. Let $*$ be a conjunction according to Definition 1. If $\exists a \neq F$ such that $T * a=F$ then $T \rightarrow_{\mathcal{R}(*)} F \neq F$.

Proof. By Equation (1d) (residuation), we have that $T \rightarrow_{\mathcal{R}(*)} F \geq a>F$.

Nevertheless, if from these implications we compute the corresponding conjunctions by $\mathcal{S}$ we obtain the connectives in Table 6 .

TABLE 6. Operation $\mathcal{S}$ applied to the residuum of conjunctions $*_{6}$ and $*_{7}$.

\begin{tabular}{c|c|c|c}
$\mathcal{S}\left(\mathcal{R}\left(*_{6}\right)\right)$ & $\mathrm{F}$ & $\mathrm{N}$ & $\mathrm{T}$ \\
\hline $\mathrm{F}$ & $\mathrm{F}$ & $\mathrm{F}$ & $\mathrm{F}$ \\
\hline $\mathrm{N}$ & $\mathrm{F}$ & $\mathrm{N}$ & $\mathrm{N}$ \\
\hline $\mathrm{T}$ & $\mathrm{F}$ & $\mathrm{N}$ & $\mathrm{N}$
\end{tabular}

\begin{tabular}{c|c|c|c}
$\mathcal{S}\left(\mathcal{R}\left(*_{7}\right)\right)$ & $\mathrm{F}$ & $\mathrm{N}$ & $\mathrm{T}$ \\
\hline $\mathrm{F}$ & $\mathrm{F}$ & $\mathrm{F}$ & $\mathrm{F}$ \\
\hline $\mathrm{N}$ & $\mathrm{F}$ & $\mathrm{F}$ & $\mathrm{N}$ \\
\hline $\mathrm{T}$ & $\mathrm{F}$ & $\mathrm{N}$ & $\mathrm{N}$
\end{tabular}

Note that the range of these operations is $\{F, N\}$ only. In fact they are $\wedge$ $\left(\mathcal{S}\left(\mathcal{R}\left(*_{6}\right)\right)\right)$ and Łukasiewicz conjunction $\left(\mathcal{S}\left(\mathcal{R}\left(*_{7}\right)\right)\right)$, but for $T * T=N$. Now, with these operations, we note that the diagrams can commute again: 

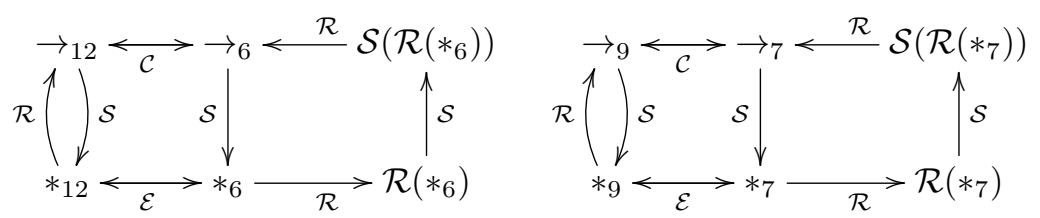

In the above patterns, we have a noticeable relationship between Sette implication (n.6) and Bochvar external one (n.12). Moreover, we can define Sette conjunction (n.1) by means of Bochvar implication (n.12) and Bochvar conjunction (n.8) through Sette implication (n.6).

$$
p *_{1} q=\neg\left(\left(\neg p \rightarrow_{12} \neg q\right) \rightarrow_{12} \neg q\right) ; \quad p *_{8} q=\neg\left(\left(\neg p \rightarrow_{6} \neg q\right) \rightarrow_{6} \neg q\right) .
$$

We also note that the converse relationships between operations $6 / 8$ and $1 / 12$ hold:

$$
p *_{12} q=\neg\left(\left(\neg p \rightarrow_{1} \neg q\right) \rightarrow_{1} \neg q\right) ; \quad p *_{6} q=\neg\left(\left(\neg p \rightarrow_{8} \neg q\right) \rightarrow_{8} \neg q\right) .
$$

To the best of our knowledge, the relationship between Sette and Bochvar implications has not been put forward previously and it deserves some further investigation. Implication $\rightarrow_{6}$ has been studied as a paraconsistent system [Sette(1973)] $\left(\rightarrow_{6}, *_{1},+_{1},-\right)$ where $+_{1}$ is the disjunction defined as:

$$
x+{ }_{1} y:= \begin{cases}F & \text { if } x=y=F \\ T & \text { otherwise }\end{cases}
$$

and designated values are $N$ and $T$. It has the property that it cannot be strengthened, i.e., there is no propositional calculus between this system and classical propositional logic. Bochvar system is defined as $\left(\rightarrow_{12}, *_{8},+_{8},-\right)$ where, likewise,

$$
x+{ }_{8} y:= \begin{cases}T & \text { if } x=T \text { or } y=T \\ F & \text { otherwise }\end{cases}
$$

It is part of a more complex framework, where two logics co-exist, an internal one where statements can be true, false or meaningless and the external one which takes into account only meaningful (that is, true or false) statements. Finally, we note that Nelson implication (n.9) is used in Nelson logic, a system $\left(\rightarrow_{9}, *_{5}, \vee, \neg,-\right)$ with two negations designed to differentiate between refutations by counter-examples and reductio ad absurdum [Nelson(1949), Vakarelov(1977)]. As discussed by Vakarelov [Vakarelov $(2005)]$, on three values it is equivalent to Lukasiewicz logic $\left(\rightarrow_{11}, \neg\right)$ through the following identities:

$$
\begin{aligned}
p \rightarrow_{L} q & =\left(p \rightarrow_{N} q\right) \wedge\left(\neg q \rightarrow_{N} \neg p\right) \\
p \rightarrow_{N} q & =p \rightarrow_{L}\left(p \rightarrow_{L} q\right) \\
-p & =p \rightarrow_{L} \neg p
\end{aligned}
$$

3.4. Class 4: implication 8. In the last case, implication $n .8$ and Bochvar conjunction are stand-alone ones, featuring the same difficulty with the residuation of the conjunction.

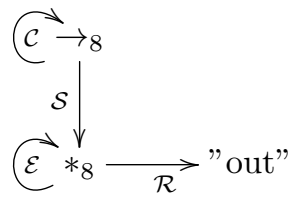


Both Bochvar conjunction and implication (n.12) can be seen as Boolean connectives through the collapse of the truth values $T$ and $\mathrm{N}$.

The pseudo-implication obtained by $\mathcal{R}\left(*_{8}\right)$ is strictly linked to Walker conjunctions. Indeed, once we consider the implications obtained by residuation of the six Walker conjunctions in Table 2, we get Sobociński implication from conjunctions $*_{W}^{3}$ and $*_{W}^{7}$, Gödel implication from $*_{W}^{6}$ and from the other three conjunctions, namely $*_{W}^{2}, *_{W}^{4}, *_{W}^{8}$ we have the implication $\rightarrow_{W}$ given in Table 7 .

TABLE 7. Implication obtained from conjunctions $*_{W}^{2}, *_{W}^{4}, *_{W}^{8}$ by residuation and derived connectives.

\begin{tabular}{c|c|c|c}
$\rightarrow_{W}$ & $\mathrm{~F}$ & $\mathrm{~N}$ & $\mathrm{~T}$ \\
\hline $\mathrm{F}$ & $\mathrm{T}$ & $\mathrm{T}$ & $\mathrm{T}$ \\
\hline $\mathrm{N}$ & $\mathrm{T}$ & $\mathrm{T}$ & $\mathrm{T}$ \\
\hline $\mathrm{T}$ & $\mathrm{N}$ & $\mathrm{N}$ & $\mathrm{T}$
\end{tabular}

\begin{tabular}{c|c|c|c}
$\mathcal{C}\left(\rightarrow_{W}\right)$ & $\mathrm{F}$ & $\mathrm{N}$ & $\mathrm{T}$ \\
\hline $\mathrm{F}$ & $\mathrm{T}$ & $\mathrm{T}$ & $\mathrm{T}$ \\
\hline $\mathrm{N}$ & $\mathrm{N}$ & $\mathrm{T}$ & $\mathrm{T}$ \\
\hline $\mathrm{T}$ & $\mathrm{N}$ & $\mathrm{T}$ & $\mathrm{T}$
\end{tabular}

\begin{tabular}{c|c|c|c}
$\mathcal{S}\left(\rightarrow_{W}\right)$ & $\mathrm{F}$ & $\mathrm{N}$ & $\mathrm{T}$ \\
\hline $\mathrm{F}$ & $\mathrm{F}$ & $\mathrm{F}$ & $\mathrm{F}$ \\
\hline $\mathrm{N}$ & $\mathrm{F}$ & $\mathrm{F}$ & $\mathrm{F}$ \\
\hline $\mathrm{T}$ & $\mathrm{F}$ & $\mathrm{N}$ & $\mathrm{N}$
\end{tabular}

\begin{tabular}{c|c|c|c}
$\mathcal{S}\left(\mathcal{C}\left(\rightarrow_{W}\right)\right)$ & $\mathrm{F}$ & $\mathrm{N}$ & $\mathrm{T}$ \\
\hline $\mathrm{F}$ & $\mathrm{F}$ & $\mathrm{F}$ & $\mathrm{F}$ \\
\hline $\mathrm{N}$ & $\mathrm{F}$ & $\mathrm{F}$ & $\mathrm{N}$ \\
\hline $\mathrm{T}$ & $\mathrm{F}$ & $\mathrm{F}$ & $\mathrm{N}$
\end{tabular}

Implication $\rightarrow_{W}$ is exactly the residuation of Bochvar conjunction $\left(*_{8}\right)$, marked as "out" in the above diagram. Moreover, from $\rightarrow_{W}$ we can define by contraposition another implication and then two other conjunctions by transformation $\mathcal{S}$. Finally, by applying again residuation to $\mathcal{S}\left(\rightarrow_{w}\right)$ we get implication $\mathrm{n} .8$ and from $\mathcal{S}\left(\mathcal{C}\left(\rightarrow_{w}\right)\right)$ we go back to $\mathcal{C}\left(\rightarrow_{W}\right)$. So, we can see that the diagram involving implication $\rightarrow_{8}$ and Bochvar conjunction, can now be completed with a diagram of the same structure as for class 2 .

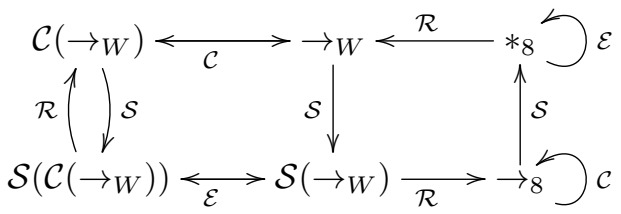

Of course, it should be noted that the semantics of the operations in Table 7 is problematic. For instance, the two implications are such that $T \rightarrow F \neq F$ and the two conjunctions are never true.

\section{Reducibility of All Connectives to one LOGiC}

We have seen that the 28 conjunction and implication connectives can be clustered into 8 groups and inside each group we can define each connective starting from one of the others. In this section, we try to discover connections among these groups. First, we remark that beyond the transformations in equations (1) we have seen in equations (2) and (3) that there are other ways to define an implication $\rightarrow_{\text {new }}$ given another implication $\rightarrow$. Namely:

$$
\begin{aligned}
& p \rightarrow_{\text {new }} q=(p \rightarrow q) \wedge(\neg q \rightarrow \neg p) ; \\
& p \rightarrow_{\text {new }} q=q \vee(p \rightarrow q) ; \\
& p \rightarrow_{\text {new }} q=p \rightarrow(p \rightarrow q) ;
\end{aligned}
$$


and others can also be generated in a similar way:

$$
\begin{aligned}
& p \rightarrow_{\text {new }} q=(p \rightarrow q) \vee(\neg q \rightarrow \neg p) ; \\
& p \rightarrow_{\text {new }} q=\neg p \vee(p \rightarrow q) .
\end{aligned}
$$

If we systematically apply them to our 14 implications, we can see that we can link all the 8 groups as outlined in Figure 1. In this graph, every circle represents a group

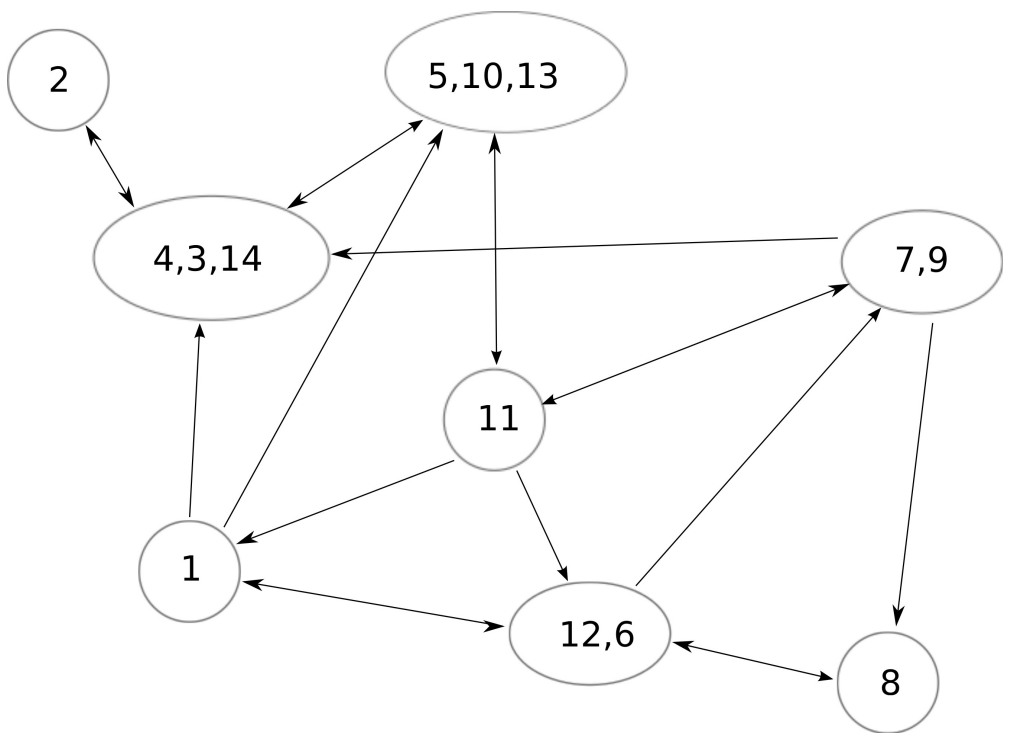

Figure 1. Outline of all the relations among connectives.

of conjunctions/implications related via transformations defined in the previous section, and an arrow from one group to another indicate that the connectives in the latter can be derived from the connectives in the former. In more details, the links we can obtain among implications with the above transformations are summarized as in Table 8, (while the transformations inside the same group are omitted).

Further, we have already shown in Remark 4 how to obtain Sette implication and conjunction from Łukasiewicz implication and also the relationship existing among conjunction/implication 1-12 and 6-8 in section 3.3. From all these connections, we note, in particular, the relationship between Kleene (n.5) and Jaśkowski implication (n.4), Bochvar (n.12) and Nelson (n.9), and Gödel (n.10) and Łukasiewicz (n.11). The significance of all these relationships deserves some further study both from the semantic and formal logic standpoint.

As a consequence, we can see that from any operation of any group, it is possible to define all the other connectives, provided that we suppose our set $\mathbf{3}$ to be ordered, hence to be equipped with $\min (\wedge), \max (\vee)$ as well as to allow the use of residuation and the involutive negation. In particular, we can start from very simple structures and show that all the connectives are definable in such structures. 
D. CIUCCI* AND D. DUBOIS ${ }^{O}$

TABLE 8. Relations among implications

\begin{tabular}{|c|c|c|}
\hline n. & how it can be obtained & name \\
\hline 1 & & \\
\hline 2 & $\left(p \rightarrow_{3} q\right) \wedge\left(\neg q \rightarrow_{3} \neg p\right),\left(p \rightarrow_{4} q\right) \wedge\left(\neg q \rightarrow_{4} \neg p\right)$ & Sobociński \\
\hline 3 & $\neg p \vee\left(p \rightarrow_{2} q\right)$ & \\
\hline 4 & $q \vee\left(p \rightarrow_{1} q\right), q \vee\left(p \rightarrow_{2} q\right)$ & Jaśkowski \\
\hline 5 & $\begin{array}{c}\neg p \vee\left(p \rightarrow_{1} q\right),\left(p \rightarrow_{3} q\right) \vee\left(\neg q \rightarrow_{3} \neg p\right), q \vee\left(p \rightarrow_{3} q\right) \\
\left(p \rightarrow_{4} q\right) \vee\left(\neg q \rightarrow_{4} \neg p\right), \neg p \vee\left(p \rightarrow_{4} q\right)\end{array}$ & (strong) Kleene \\
\hline 6 & & Sette \\
\hline 7 & $\neg p \vee\left(p \rightarrow_{6} q\right)$ & \\
\hline 8 & $\begin{array}{c}\left(p \rightarrow_{6} q\right) \vee\left(\neg q \rightarrow_{6} \neg p\right),\left(p \rightarrow_{7} q\right) \vee\left(\neg q \rightarrow_{7} \neg p\right) \\
p \rightarrow_{7}\left(p \rightarrow_{7} q\right),\left(p \rightarrow_{9} q\right) \vee\left(\neg q \rightarrow_{9} \neg p\right) \\
\left(p \rightarrow_{12} q\right) \vee\left(\neg q \rightarrow_{12} \neg p\right)\end{array}$ & \\
\hline 9 & $p \rightarrow_{11}\left(p \rightarrow_{11} q\right), q \vee\left(p \rightarrow_{12} q\right)$ & Nelson \\
\hline 10 & $q \vee\left(p \rightarrow_{14} q\right)$ & Gödel \\
\hline 11 & $\begin{array}{c}\left(p \rightarrow_{7} q\right) \wedge\left(\neg q \rightarrow_{7} \neg p\right),\left(p \rightarrow_{9} q\right) \wedge\left(\neg q \rightarrow_{9} \neg p\right) \\
\left(p \rightarrow_{10} q\right) \vee\left(\neg q \rightarrow_{10} \neg p\right), \neg p \vee\left(p \rightarrow_{10} q\right), \\
\left(p \rightarrow_{13} q\right) \vee\left(\neg q \rightarrow_{13} \neg p\right), p \vee\left(p \rightarrow_{13} q\right)\end{array}$ & Łukasiewicz \\
\hline 12 & $p \rightarrow_{12} q=p \rightarrow_{13}\left(p \rightarrow_{13} q\right)$ & Bochvar external \\
\hline 13 & $\neg p \vee\left(p \rightarrow_{14} q\right)$ & \\
\hline 14 & $\begin{aligned}\left(p \rightarrow_{6} q\right) & \wedge\left(\neg q \rightarrow_{6} \neg p\right),\left(p \rightarrow_{10} q\right) \wedge\left(\neg q \rightarrow_{10} \neg p\right) \\
\left(p \rightarrow_{12} q\right) & \wedge\left(\neg q \rightarrow_{12} \neg p\right),\left(p \rightarrow_{13} q\right) \wedge\left(\neg q \rightarrow_{13} \neg p\right)\end{aligned}$ & Gaines-Rescher \\
\hline
\end{tabular}

Proposition 7. Let 3 be the three-element set with the usual order $F<N<T$ or equivalently, $\mathbf{3}=(\overline{3}, \wedge, \vee)$, with $\overline{3}$ the set of three elements without the order structure. All the 14 conjunctions and implications can be defined in any of the following systems:

- $\left(\mathbf{3}, \neg, \rightarrow_{10}\right)=\left(\overline{3}, \wedge, \neg, \rightarrow_{10}\right)$;

- $\left(\mathbf{3}, \rightarrow_{i}\right)=\left(\overline{3}, \wedge, \vee, \rightarrow_{i}\right)$ where $i \in I=\{3,5,7,11,13\}$.

Proof. The proof is straigthforward from the above discussion, the relationships outlined inside the groups and among them, and the fact that from all implications $\rightarrow_{i}, i \in I$ we can recover the involutive negation as $\neg p=p \rightarrow_{i} 0$.

Note that the first item of the above proposition holds for any system $\left(\mathbf{3}, *_{i}, \neg\right)$, $\left(\mathbf{3}, \rightarrow_{i}, \neg\right)$. Indeed, it is possible to obtain all other connectives from any implication and any conjunction provided we have the order relation and the involutive negation. On the other hand, in some cases, we can even simplify the starting structure, since it is possible to obtain the order from the implication.

Proposition 8. We denote by $\overline{3}$ the set of three elements without any structure. All the 14 conjunctions and implications can be defined in any of the following systems (letting $F$ be understood as a unary function always yielding $F$ ) :

- $\left(\overline{3}, \rightarrow_{11}, F\right)$ where $\rightarrow_{11}$ is Eukasiewicz implication;

- $\left(\overline{3}, \rightarrow_{9}, \neg\right)$ where $\rightarrow_{9}$ is Nelson implication;

- $\left(\overline{3}, \rightarrow_{5}, \sim\right)$ where $\rightarrow_{5}$ is Kleene implication and $\sim$ the intuitionistic negation.

Proof. First, we see that the constant function $F$ is definable in Kleene logic through the intuitionistic negation as $\sim\left(p \rightarrow_{5} p\right)$ and that the involutive negation is definable 
in Eukasiewicz and Kleene systems as $\neg p:=p \rightarrow_{11} F=p \rightarrow_{5} F$. Then, the second and third cases are reducible to the first one since

- in Nelson logic, Łukasiewicz implication is $a \rightarrow_{11} b:=\left(p \rightarrow_{9} q\right) \wedge\left(\neg q \rightarrow_{9}\right.$ $\neg p)$ and $F:=\neg\left(p \rightarrow_{11} p\right)$;

- in Kleene logic, the Eukasiewicz implication can be defined as $a \rightarrow_{11} b:=$ $(\neg \sim \neg a \vee b) \wedge(\neg \sim b \vee \neg a)$.

Now, we prove the proposition for the first case (and consequently for the other two). From Eukasiewicz we can obtain Nelson implication as $p \rightarrow_{11}\left(p \rightarrow_{11} q\right)$ and consequently paraconsistent negation - . Then, Gödel implication is $p \rightarrow_{10} q:=-\left(p \rightarrow_{11}\right.$ $q) \rightarrow_{11} q$ and then, we can obtain intuitionistic negation and implication $\rightarrow_{13}$. From Nelson implication we arrive at implication $\rightarrow_{7}$ and from Gödel implication we also obtain Gaines-Rescher $p \rightarrow_{14} q=\left(p \rightarrow_{10} q\right) \wedge\left(\neg q \rightarrow_{10} \neg p\right)$. Then, we get (see Class 2 in the previous section) implications 3 and 4 (Jaśkowski) and from 4 , we reach Sobociński implication $\rightarrow_{2}$. Bochvar implication is $p \rightarrow_{12} q:=p \rightarrow_{13}\left(p \rightarrow_{13} q\right)$ and then we get Sette implication $\rightarrow_{6}$. Finally, from $\rightarrow_{6}$ we obtain Sette conjunction $p *_{1} q=\left(\left(\left(p \rightarrow_{6} p\right) \rightarrow_{6} p\right) \rightarrow_{6}-\left(\left(q \rightarrow_{6} q\right) \rightarrow_{6} q\right)\right) \rightarrow_{6}-\left(p \rightarrow_{6}-q\right)$ and so implication $\rightarrow_{1}$. Finally, implication $\rightarrow_{8}$ is definable for instance as $p \rightarrow_{7}\left(p \rightarrow_{7} q\right)$ and then we get also Bochvar conjunction $*_{8}$.

Let us note that in order to obtain implications $1,2,3$, 4 , we have to apply residuation at some point. Moreover, in the last case of the proposition, the intuitionistic negation $\sim$ can be replaced by a paraconsistent one -, as they are mutually definable.

The reader may question the significance of the results in this section. As said earlier, on the one hand, we may consider that three-valued logic looks as basic to human reasoning as Boolean logic, in the sense that it relies on the most elementary bipolar scale, and that bipolarity is known to be a basic feature in the study of cognitive processes (See [Dubois and Prade(2008)] and the special issue containing this paper). Moreover, many interpretations of the third truth-value listed in the introduction have been proposed in intuitively natural contexts. Most of them (except for the case of undefined) are consistent with the conditions posed here to define implications and conjunctions, namely monotonicity and reduction to classical logic on $\{T, F\}$. While each of these interpretations may lead us to define specific three-valued connectives, what is proved here is that we always obtain fragments of a single three-valued algebraic structure, where all 28 connectives (and three negations) can be obtained from very few of them. Especially, the structure defined by means of Lukasiewicz implication seems to cover them all. Hence, instead of viewing three-valued logics as different unrelated structures, we are led to consider each of them as a fragment of a unique larger structure (e.g. a three-valued MV-algebra). Note that a similar role is given to Boolean algebras in the two-valued case : one may consider the fragment obtained by conjunction and disjunction only, or the structure based on exclusive or. Still, Boolean algebra appears as the unified framework. Our paper suggests that the same unique structure can account for three-valued truth-tables extending Boolean ones in a consistent manner, which may potentially be useful for unifying the various three-valued systems. 


\section{RELATIONSHIP WITH FUNCTIONAL COMPLETENESS}

It is known since Rosser and Turquette [Rosser and Turquette(1952)] that any binary connective can be defined in Eukasiewicz logic provided the third value $N$ is included in the language. They proved the following result:

Proposition 9. Let $\mathcal{L}=\left(\overline{3}, \rightarrow_{11}, F, N\right)$ be three-valued Lukasiewicz logic plus the value $N$. Then, any binary connective can be defined in $\mathcal{L}$.

They establish this proposition for $n$-valued Łukasiewicz logic, where $N$ is truthvalue next to $T$. Their proof is complex, and we give a simpler account of this result below, in the three-valued case. First, we can define the following operations

$$
\begin{aligned}
\neg p & =p \rightarrow_{11} F & & \text { involutive negation } \\
p \oplus q & =\neg p \rightarrow_{11} q & & \text { bounded sum } \\
p \odot q & =\neg(\neg p \oplus \neg q) & & \text { Eukasiewicz conjunction } \\
p \vee q & =\left(p \rightarrow_{11} q\right) \rightarrow_{11} q & & \text { lattice disjunction } \\
p \wedge q & =\neg(\neg p \vee \neg q) & & \text { lattice conjunction } \\
\nabla p & =\neg p \rightarrow_{11} p=p \oplus p & & \text { possibility } \\
\Delta p & =\neg(\nabla \neg p)=p \odot p & & \text { necessity }
\end{aligned}
$$

Now, for each of the nine possible combinations of two values $x, y \in \overline{3}$, we define a function $c_{x, y}^{T}(p, q)$ which takes value $T$ on $x, y$ and $F$ otherwise. Then, we can define a function which instead of $T$ takes value $N$ as $c_{x, y}^{N}(p, q):=N \odot c_{x, y}^{T}(p, q)$. Finally, we can sum, either with $\oplus$ or with $\vee$, these functions to obtain $T$ or $N$ where desired and $F$ otherwise. The functions $c_{x, y}^{T}$ are defined in Table 9.

TABLE 9. Table defining function $c_{x, y}^{T}$, missing values are obtained by commutativity.

\begin{tabular}{c|c|c|c}
$c_{x, y}^{T}$ & $\mathrm{~F}$ & $\mathrm{~N}$ & $\mathrm{~T}$ \\
\hline $\mathrm{F}$ & $\Delta(\neg p \odot \neg q)$ & & \\
\hline $\mathrm{N}$ & $(\nabla(p) \wedge \nabla(\neg p)) \odot \Delta(\neg q)$ & {$[\nabla p \wedge \nabla(\neg p)] \odot[\nabla q \wedge \nabla(\neg q)]$} & \\
\hline $\mathrm{T}$ & $\Delta(p \odot \neg q)$ & $\Delta p \odot[\nabla q \wedge \nabla(\neg q)]$ & $\Delta(p \odot q)$
\end{tabular}

Finally, the function equal to $F$ for all $x, y$ is obtained by the conjunction of any two functions $c_{x, y}^{T}$.

Example 1. Let us consider two conjunctions, which we already know to not satisfy Definition 1: weak Kleene and McCarty conjunctions. In the case of weak Kleene logic, the interpretation of the third value is undefined and the conjunction truth table is given in Table 10.

TABLE 10. Weak Kleene conjunction

\begin{tabular}{c|c|c|c}
$*_{w K}$ & $\mathrm{~F}$ & $\mathrm{~N}$ & $\mathrm{~T}$ \\
\hline $\mathrm{F}$ & $\mathrm{F}$ & $\mathrm{N}$ & $\mathrm{F}$ \\
\hline $\mathrm{N}$ & $\mathrm{N}$ & $\mathrm{N}$ & $\mathrm{N}$ \\
\hline $\mathrm{T}$ & $\mathrm{F}$ & $\mathrm{N}$ & $\mathrm{T}$
\end{tabular}


It can be defined in Eukasiewicz logic $+N$ in accordance with the proof of the above proposition as:

$$
\begin{aligned}
& N \odot[(\nabla(p) \wedge \nabla(\neg q)) \odot \Delta(\neg p)] \vee \\
& N \odot[(\nabla(p) \wedge \nabla(\neg p)) \odot \Delta(\neg q)] \vee \\
& N \odot[(\nabla p \wedge \nabla(\neg p)) \odot(\nabla q \wedge \nabla(\neg q))] \vee \\
& N \odot[\Delta q \odot(\nabla p \wedge \nabla(\neg p))] \vee \\
& N \odot[\Delta p \odot(\nabla q \wedge \nabla(\neg q))] \vee \\
& {[\Delta(p \odot q)]}
\end{aligned}
$$

or more simply as:

$$
N \odot[(\nabla(q) \wedge \nabla(\neg q)) \vee(\nabla p \wedge \nabla(\neg p))] \vee \Delta(p \odot q) .
$$

In the theory of computation by McCarthy, $N$ is the value given to a process that does not terminate. As such, if in the conjunction $p *_{M C} q$, we first try to compute $p$ and it does not terminate, then also the value of the whole proposition remains undefined. However, if $p=F$ then the conjunction is false irrespective of the value of $q$. This process motivates the non-commutative conjunction in Table 11 .

TABLE 11. McCarthy conjunction

\begin{tabular}{c|c|c|c}
$*_{M C}$ & $\mathrm{~F}$ & $\mathrm{~N}$ & $\mathrm{~T}$ \\
\hline $\mathrm{F}$ & $\mathrm{F}$ & $\mathrm{F}$ & $\mathrm{F}$ \\
\hline $\mathrm{N}$ & $\mathrm{N}$ & $\mathrm{N}$ & $\mathrm{N}$ \\
\hline $\mathrm{T}$ & $\mathrm{F}$ & $\mathrm{N}$ & $\mathrm{T}$
\end{tabular}

Its definition in Lukasiewic logic $+N$ is as follows ${ }^{10}$ :

$$
\begin{aligned}
& N \odot[(\nabla p \wedge \nabla(\neg p)) \odot \Delta(\neg q)] \vee \\
& N \odot[(\nabla p \wedge \nabla(\neg p)) \odot(\nabla q \wedge \nabla(\neg q))] \vee \\
& N \odot[\Delta q \odot(\nabla p \wedge \nabla(\neg p))] \vee \\
& N \odot[\Delta p \odot[\nabla q \wedge \nabla(\neg q)] \vee \\
& \Delta(p \odot q)
\end{aligned}
$$

or as $N \odot[(\nabla p \wedge \nabla(\neg p)) \vee \Delta p \odot(\nabla q \wedge \nabla(\neg q))] \vee \Delta(p \odot q)$.

In [Jobe(1962)], it is proved that in a many-valued logic with $\wedge$ and $\vee$ it is enough to have functional completeness for unary operators in order to have functional completeness for $n$-ary operators with $n \geq 2$. Once we are able to prove the unary completeness, the author gives a constructive procedure to build any other function (Lemma 1 in [Jobe(1962)]). Then, the author proposes a notion of normal form understood as a formula which contains $\wedge, \vee$ and unary operators, with no binary operators in the scope of a unary one and no disjunction $(V)$ in the scope of a conjunction $(\wedge)$. Now, while Eukasiewicz logic plus the constant $N$ is functionally

\footnotetext{
${ }^{10}$ For the sake of completeness we note that from residuation of both the conjunctions $* w K$ and $*_{M C}$ we get Gödel implication.
} 
complete, it is not canonically complete, that is, not any function can be defined using only formulas in normal form. However, if we add the necessity operator, i.e., we use $(\wedge, \vee, \neg, N, \Delta)$, canonical completeness is obtained:

Proposition 10. Let $\mathcal{L}=\left(\overline{3}, \rightarrow_{11}, \neg, N, \Delta\right)$ be three-valued Lukasiewicz logic plus the value $N$ and necessity operator $\Delta$. Then, any unary connective can be defined in $\mathcal{L}$ in a normal form.

Proof. It is enough to show that we can represent in normal form the three functions of the following table

\begin{tabular}{c|c|c|c}
$p$ & $J_{T}^{1}(p)$ & $J_{T}^{2}(p)$ & $J_{T}^{3}(p)$ \\
\hline $\mathrm{F}$ & $\mathrm{F}$ & $\mathrm{F}$ & $\mathrm{T}$ \\
\hline $\mathrm{N}$ & $\mathrm{F}$ & $\mathrm{T}$ & $\mathrm{F}$ \\
\hline $\mathrm{T}$ & $\mathrm{T}$ & $\mathrm{F}$ & $\mathrm{F}$
\end{tabular}

Now, $J_{T}^{1}(p)=\Delta(p), J_{T}^{2}(p)=\neg \Delta(\neg p) \wedge \neg \Delta(p)$ and $J_{T}^{3}(p)=\Delta(\neg p)$.

Now, using the above result and according to [Jobe(1962), Lemma 1], we can build a table similar to Table 9 as

\begin{tabular}{c|c|c|c}
$c_{x, y}^{T}$ & $\mathrm{~F}$ & $\mathrm{~N}$ & $\mathrm{~T}$ \\
\hline $\mathrm{F}$ & $\Delta(\neg p) \wedge \Delta(\neg q)$ & & \\
\hline $\mathrm{N}$ & $\neg \Delta(\neg p) \wedge \neg \Delta p \wedge \Delta(\neg q)$ & $\neg \Delta(\neg p) \wedge \neg \Delta p \wedge \neg \Delta(\neg q) \wedge \neg \Delta q$ & \\
\hline $\mathrm{T}$ & $\Delta p \wedge \Delta(\neg q)$ & $\Delta p \wedge \neg \Delta(\neg q) \wedge \neg \Delta q$ & $\Delta p \wedge \Delta q$
\end{tabular}

In the previous sections, we focused on three-valued logics extending classical logic, that is, whose truth tables coincide with Boolean connectives for Boolean entries. We can call extended Boolean function, any such function $f: \mathbf{3}^{n} \rightarrow \mathbf{3}$ whose restriction to $\{T, F\}$ ranges on $\{T, F\}$. From the fact that Eukasiewicz logic is meant to encode extended Boolean functions only, it is impossible to define in it any function which violates this restriction. The fact that in Łukasiewicz logic we can represent only extended Boolean functions is valid for any number of truth values (and for any set of designated values). Indeed, this was already observed by Rosser and Turquette [Rosser and Turquette(1952)] in their book. After defining functions $J_{k}(p)=T$ if $k=p$ and 0 otherwise for all truth values $k$, they observe that any statement which is built using only $\rightarrow$ and $\neg$ is such that its corresponding truth function $f\left(x_{1}, \ldots x_{n}\right)$ always takes the value $\mathrm{T}$ or $\mathrm{F}$ if only the values $\mathrm{T}$ or $\mathrm{F}$ are assigned to $x_{1}, \ldots, x_{n}$. And in order to obtain functional completeness they are obliged to add the constant function next to $T$ (here $N$ ) as an extra primitive.

All extended Boolean functions are of course definable by Propositions 9 or 10 . If the value $N$ must be obtained when one of the argument, call it $p$, has value $N$, then it is enough to form the conjunction with $p$ in the desired position. For instance, to define a binary function $f(p, q)$ such that $f(T, N)=N$ it is sufficient, according to Prop. 10, to consider the function $[\Delta(p) \wedge \neg \Delta(q) \wedge \neg \Delta(\neg q)] \wedge q$ which exactly returns $N$ when the input is $(T, N)$ and $F$ otherwise. However our results in the paper differ from functional completeness in the above sense, as we establish the possibility, in the three-valued case, to generate all extended Boolean functions from a few elementary such functions, showing the unity of the formal framework for three-valued extensions of classical logic. 


\section{Conclusion}

This paper is a preliminary attempt to clarify the connections between threevalued logics extending classical logic, from a truth-table point of view. As we have seen, basic connectives such three-valued systems are mathematically connected together and they can all be defined starting from very simple algebraic structures.

On this basis, these relationships should be exploited with respect also to their significance in knowledge representation in order to better understand which connectives make more sense in reference to the intended meaning of the third value, as reviewed in the introduction. In this way, we will be able to relate as many three-valued calculi as possible to a class of applications where the third truthvalue makes sense. This contrasts with many previous studies whose focus was essentially syntactic and proof-theoretic. The latter point of view is clearly instrumental for mechanizing three-valued reasoning tasks, but such machineries need sound appropriate canonical examples to be accepted and properly applied.

More recently, we have studied a case when an epistemic flavour is attached to the third value, interpreted as unknown, thus referring to the idea of lack of knowledge. In this case, a translation from the three-valued logic framework into an adequate epistemic logic setting encapsulating classical propositional logic has been provided, namely the subjective fragment of S5 modal logic with no nested modality [Banerjee and Dubois(2009)]. First results [Ciucci and Dubois(2012b)] indicate that Łukasiewicz logic is equivalent to a fragment of this modal logic, where modalities only apply to litterals. They suggest that any three-valued extension of classical propositional logic as studied in the present paper can be captured by this modal fragment, which lays bare the intuitive meaning of three-valued formulas when the third truth-value refers to the idea of unknown. It offers a bridge between epistemic logics and three-valued logics of incomplete information. Other translations of our three-valued setting can be envisaged, properly choosing the target language, for other understandings of the third truth-value.

\section{REFERENCES}

[Adamatzky(2003)] Adamatzky, A., 2003. On dynamically non-trivial three-valued logics. Chaos, Solitons and Fractals 18, 917-936.

[Asenjo and Tamburino(1975)] Asenjo, F. G., Tamburino, J., 1975. Logic of antinomies. Notre Dame journal of formal logic 16, 17-44.

[Avron(1986)] Avron, A., 1986. On An Implication Connective of RM. Notre Dame Journal of Formal Logic 27, 201-209.

[Baaz(1996)] Baaz, M., 1996. Infinite-valued Gödel Logics with 0-1 Projections and Relativizations. In: Hájek, P. (Ed.), GÖDEL96-Logical Foundations of Mathematics, Computer Science and Physics. Vol. 6 of Lecture Notes in Logic. Springer-Verlag, Berlin, pp. 23-33.

[Baets and Fodor(1999)] Baets, B. D., Fodor, J. C., 1999. Residual operators of uninorms. Soft Computing 3, 89-100.

[Banerjee and Dubois(2009)] Banerjee, M., Dubois, D., 2009. A simple modal logic for reasoning about revealed beliefs. In: Sossai, C., Chemello, G. (Eds.), Proc. ECSQARU 2009, Verona, Italy, LNAI 5590. Springer-Verlag, pp. 805-816.

[Belluce(1997)] Belluce, L. P., 1997. Generalized fuzzy connectives on MV-algebras. J. Math. Anal. Appl. 206, 485-499.

[Belnap(1977)] Belnap, N. D., 1977. A useful four-valued logic. In: Dunn, J. M., Epstein, G. (Eds.), Modern Uses of Multiple-Valued Logic. D. Reidel, pp. 8-37.

[Bochvar(1981)] Bochvar, D. A., 1981. On a Three-Valued Logical Calculus and its Application to the Analysis of the Paradoxes of the Classical Extended Functional Calculus. History and Philosophy of Logic 2, 87-112. 
[Borowski(1970)] Borowski, L. (Ed.), 1970. Selected works of J. Łukasiewicz. North-Holland, Amsterdam.

[Cattaneo and Ciucci(2003)] Cattaneo, G., Ciucci, D., 2003. Shadowed sets and related algebraic structures. Fundamenta Informaticae 55, 255-284.

[Cattaneo et al.(2004a)Cattaneo, Ciucci, Giuntini, and Konig] Cattaneo, G., Ciucci, D., Giuntini, R., Konig, M., 2004a. Algebraic Structures Related to Many Valued Logical Systems. Part I: Heyting Wajsberg Algebras. Fundamenta Informaticae 63 (4), 331-355.

[Cattaneo et al.(2004b)Cattaneo, Ciucci, Giuntini, and Konig] Cattaneo, G., Ciucci, D., Giuntini, R., Konig, M., 2004b. Algebraic Structures Related to Many Valued Logical Systems. Part II: Equivalence Among some Widespread Structures. Fundamenta Informaticae 63 (4), $357-373$.

[Cattaneo et al.(1999)Cattaneo, Giuntini, and Pilla] Cattaneo, G., Giuntini, R., Pilla, R., 1999. $\mathrm{BZMV}^{d M}$ and Stonian MV algebras (applications to fuzzy sets and rough approximations). Fuzzy Sets and Systems 108, 201-222.

[Cignoli(1986)] Cignoli, R., 1986. The class of Nelson algebras satisfying an interpolation property and Nelson algebras. Algebra Universalis 23, 262-292.

[Ciucci and Dubois(2010)] Ciucci, D., Dubois, D., 2010. Truth-Functionality, Rough Sets and Three-Valued Logics. In: Proceedings ISMVL. pp. 98-103.

[Ciucci and Dubois(2012a)] Ciucci, D., Dubois, D., 2012a. Relationships between connectives in three-valued logics. In: Proc. IPMU Conference, Catania. Vol. 297 of CCIS. Springer, pp. 633-642.

[Ciucci and Dubois(2012b)] Ciucci, D., Dubois, D., 2012b. Three-valued logics for incomplete information and epistemic logic. In: Proc. 13th European Conference on Logics in Artificial Intelligence (JELIA), Toulouse, France. Vol. 7519 of LNCS. pp. 147-159.

[Codd(1979)] Codd, E. F., 1979. Extending the database relational model to capture more meaning. ACM Trans. Database Syst. 4 (4), 397-434.

[De Baets et al.(2009)De Baets, Fodor, Ruiz-Aguilera, and Torrens] De Baets, B., Fodor, J. C., Ruiz-Aguilera, D., Torrens, J., 2009. Idempotent uninorms on finite ordinal scales. International Journal of Uncertainty, Fuzziness and Knowledge-Based Systems, 1-14.

[D'Ottaviano and da Costa(1970)] D'Ottaviano, I. M. L., da Costa, N. C. A., 1970. Sur un problème de Jaśkowski. Comptes Rendus de l'Académie des Science 270, 1349-1353.

[Drewniak(2006)] Drewniak, J., 2006. Invariant fuzzy implications. Soft Computing 10 (6), 506513.

[Dubois(2008)] Dubois, D., 2008. On Ignorance and Contradiction Considered as Truth-Values. Logic Journal of the IGPL 16, 195-216.

[Dubois and Prade(1984a)] Dubois, D., Prade, H., 1984a. Fuzzy-set-theoretic differences and inclusions and their use in the analysis of fuzzy equations. Control and Cybernetics 13 (3), 129-146.

[Dubois and Prade(1984b)] Dubois, D., Prade, H., 1984b. A theorem on implication functions defined from triangular norms. Stochastica VIII, 267-279.

[Dubois and Prade(1994)] Dubois, D., Prade, H., 1994. Conditional Objects as Nonmonotonic Consequence Relationships. IEEE Transaction of Sysyems, Man, and Cybernetics 24 (12), $1724-1740$.

[Dubois and Prade(2001)] Dubois, D., Prade, H., 2001. Possibility theory, probability theory and multiple-valued logics: A clarification. Ann. Math. and AI 32, 35-66.

[Dubois and Prade(2008)] Dubois, D., Prade, H., 2008. An introduction to bipolar representations of information and preference. Int. J. Intelligent Systems 23 (3), 866-877.

[Fitting(1985)] Fitting, M., 1985. A kripke-kleene semantics for logic programs. J. Log. Program. 2 (4), 295-312.

[Fodor(1991)] Fodor, J. C., 1991. On fuzzy implications operators. Fuzzy Sets and Systems 42, 293-300.

[Fox(1990)] Fox, J., 1990. Motivation and Demotivation of a Four-Valued Logic. Notre Dame Journal of Formal Logic 31 (1), 76-80.

[Gentilhomme(1968)] Gentilhomme, M. Y., 1968. Les ensembles flous en linguistique. Cahiers de linguistique theoretique et applique, Bucarest 47, 47-65.

[Hájek(1998)] Hájek, P., 1998. Metamathematics of Fuzzy Logic. Kluwer, Dordrecht.

[Hardegree(1981)] Hardegree, G. M., 1981. Material implication in orthomodular (and Boolean) lattices. Notre Dame Journal of Modal Logic 22, 163-182. 
[Hosoi(1996)] Hosoi, T., 1996. The axiomatization of the intermediate propositional systems Sn of Gödel. J. Coll. Sci., Imp. Univ. Tokyo 13, 183-187.

[Jaśkowski(1969)] Jaśkowski, S., 1969. Propositional calculus for contradictory deductive systems. Studia Logica 24, 143-160.

[Jobe(1962)] Jobe, W., 1962. Completeness and canonical forms in many-valued logics. The Journal of Symbolic Logic 27, 409-422.

[Kleene(1952)] Kleene, S. C., 1952. Introduction to metamathematics. North-Holland Pub. Co., Amsterdam.

[Klement et al.(2000)Klement, Mesiar, and Pap] Klement, E. P., Mesiar, R., Pap, E., 2000. Triangular Norms. Kluwer Academic, Dordrecht.

[Konikowska(1996)] Konikowska, B., 1996. McCarthy Algebras: A Model of McCarthy's Logical Calculus. Fundamenta Informaticae 26 (2), 167-203.

[Lawry and Tang(2012)] Lawry, J., Tang, Y., 2012. On truth-gaps, bipolar belief and the assertability of vague propositions. Artif. Intell. 191-192, 20-41.

[Mas et al.(1999a)Mas, Mayor, and Torrens] Mas, M., Mayor, G., Torrens, J., 1999a. t-Operators. International Journal of Uncertainty, Fuzziness and Knowledge-Based Systems 7 (1), 31-50.

[Mas et al.(1999b)Mas, Mayor, and Torrens] Mas, M., Mayor, G., Torrens, J., 1999b. t-Operators and Uninorms on a Finite Totally Ordered Set. International Journal of Intelligent Systems $14,909-922$

[Mas et al.(2004)Mas, Monserrat, and Torrens] Mas, M., Monserrat, M., Torrens, J., 2004. Simplications and R-implications on a Finite Chain. Kibernetika 40, 3-20.

[Mas et al.(2007)Mas, Monserrat, Torrens, and Trillias] Mas, M., Monserrat, M., Torrens, J., Trillias, E., 2007. A survey on Fuzzy Implication Functions. IEEE Trans. on Fuzzy Systems $15,1107-1121$.

[McCarthy(1963)] McCarthy, 1963. A basis for a mathematical theory of computation. In: Braort, P., Hirshberg, D. (Eds.), Computer Programming and Formal Systems. North-Holland.

[Nelson(1949)] Nelson, D., 1949. Constructible Falsity. J. of Symbolic Logic 14, 16-26.

[Pearce(2006)] Pearce, D., 2006. Equilibrium logic. Annals of Mathematics and Artificial Intelligence $47,3-41$.

[Pedrycz(2009)] Pedrycz, W., 2009. From fuzzy sets to shadowed sets: Interpretation and computing. Int. J. Intell. Syst. 24 (1), 48-61.

[Priest(1979)] Priest, G., 1979. The Logic of Paradox. The Journal of Philosophical Logic 8, 219241.

[Rosser and Turquette(1952)] Rosser, J., Turquette, A., 1952. Many-Valued Logics. Studies in Logic and The Foundations of Mathematics. North-Holland, Amsterdam.

[Sabo(1998)] Sabo, M., 1998. On many valued implications. Tatra Mountain Mathematical Publication 14, 161-167.

[Sette(1973)] Sette, A. M., 1973. On propositional calculus P_1. Math. Japon. 16, 173-180.

[Smets and Magrez(1987)] Smets, P., Magrez, P., 1987. Implication in Fuzzy Logic. Int. J. of Approximate Reasoning 1, 327-347.

[Smith(1981)] Smith, K. C., 1981. The prospects for multivalued logic: A technology and applications view. IEEE Trans. Computers 30 (9), 619-634.

[Sobociński(1952)] Sobociński, B., 1952. Axiomatization of a partial system of three-value calculus of propositions. J. of Computing Systems 1, 23-55.

[Urquhart(1986)] Urquhart, A., 1986. Many-Valued Logic. In: Gabbay, D. M., Guenthner, F. (Eds.), Handbook of Philosophical Logic: Volume III, Alternatives to Classical Logic. Springer.

[Vakarelov(1977)] Vakarelov, D., 1977. Notes on N-Lattices and Constructive Logic with Strong Negation. Studia Logica 36, 109-125.

[Vakarelov(2005)] Vakarelov, D., 2005. Nelson's Negation on the Base of Weaker Versions of Intuitionistic Negation. Studia Logica 80, 393-430.

[Walker(1994)] Walker, E. A., 1994. Stone Algebras, Conditional Events, and Three Valued Logic. IEEE Transaction of Systems, Man, and Cybernetics 24 (12), 1699-1707.

[Yao(2009)] Yao, Y., 2009. Interval Sets and Interval-Set Algebras. In: Proceedings of the 8th IEEE International Conference on Cognitive Informatics. pp. 307-314.

[Yao(2012)] Yao, Y., 2012. An Outline of a Theory of Three-way Decisions. In: Proceedings of RSCTC 2012. Vol. LNCS 7413. pp. 1-17. 
*Disco, Università di Milano-Bicocca, Viale Sarca 336/14, I-20126 Milano (Italy), ${ }^{o}$ IRIT, Université Paul Sabatier, 118 route de Narbonne, 31062, Toulouse, Cedex 4 (FrANCE).

E-mail address: ciucci@disco.unimib.it, dubois@irit.fr 